\title{
Scheduling for a Single-Terminal Intermodal System Recovery with Poisson Arrivals
}

\author{
Nikola Marković - Paul Schonfeld* \\ Department of Civil and Environmental Engineering, University of Maryland, College Park, USA
}

\begin{abstract}
This paper studies the recovery of an intermodal freight system from a major disruption and develops a model for optimising vehicle schedules under disrupted conditions. The proposed model optimises the recovery of a single-terminal system with relatively short feeder routes on which vehicle roundtrip times are exponentially distributed and arrivals at the terminal are Poisson-distributed. Mathematical expectations are used to formulate the deterministic equivalent for the scheduling problem and a genetic algorithm is applied to optimise the schedules on main routes. The model developed in this paper can be applied to single-terminal transfer systems with any combination of transportation modes using discrete vehicles, as long as the feeder arrivals do not deviate much from the assumed Poisson distributions. Since its computational time is relatively insensitive to the numbers of vehicles on feeder routes, this model can be used to efficiently optimise intermodal systems with numerous vehicle arrivals.
\end{abstract}

Keywords: scheduling, disruption, intermodal, Poisson, genetic algorithm, transfer

\section{O INTRODUCTION}

Efficient transfer coordination in an intermodal transportation network can reduce the dwell times of cargos at the transfer terminals where various routes interconnect, thereby also increasing the vehicle utilisation rates, reducing the need for direct routes to connect many origins and destinations, reducing storage requirements at transfer terminals, and improving total system efficiency. In this paper we analyse an intermodal freight system with a single transfer hub and develop a model that optimises the schedule of vehicles on main routes while assuming Poisson arrivals on feeder routes. This model determines the departure times on main routes that minimize the supplier's overall system cost, including storage, vehicle, in-terminal operation and late delivery penalty costs.

The optimisation problem addressed in this paper is related to some classical problems of operations research, such as machine scheduling, lot sizing, and supply chains. Somewhat related machine scheduling problems can be found in [1] to [3]. [4] to [7] address the scheduling problem in transfer systems, but under different conditions from those considered here. For example, [4] and [5] analyse different transfer coordination policies and determine the thresholds in the intermodal systems with complex multistop routes and lower variance in travel durations, both typical for normal operations. In this paper, we analyse the case with high variances in travel times, which are typical of disrupted operations and model the arrivals as a Poisson process. [6] and [7] deal with scheduling takeoff times, a problem that will be studied in this paper. Both papers optimise departures on a single airline route and under different demand assumptions from those considered here (i.e. [6] assumes uniform demand, whereas [7] adopts time dependent demand). This paper is based on the same framework as [8] and develops a model which, unlike [8], is suitable for intermodal systems with numerous arrivals of vehicles on feeder routes. In [8], Marković and Schonfeld develop a scheduling model which assumes generally distributed vehicle roundtrip durations and vehicles operating on multiple feeder routes. Low computational efficiency of the stochastic program used in [8] enabled only the optimisation of schedules in systems with relatively few arrivals on feeder routes. In this paper we provide a computationally less demanding model by assuming exponentially distributed vehicle roundtrips and fixed fleet size on feeder routes. These assumptions allow us to model the arrivals as a stationary Poisson process and derive the expectations needed to formulate a scheduling problem that is optimised much more efficiently than the stochastic program in [8]. Thus, the model developed here can efficiently optimise large intermodal systems with numerous arrivals on feeder routes.

In this paper we analyse the recovery of a system from a major disruption during which large amounts of freight have accumulated along the feeder routes, which are assumed here to be served by trucks. To dissipate the backlogs we let the trucks on feeder routes operate nonstop and deliver cargo to the terminal where the freight is transferred to main routes, which are assumed here to be aircraft routes. Thus our transfer terminal represents an airport hub. We use pre-determined fleet sizes on feeder routes and seek to optimise the number of departures and specific 
departure schedules on main (air) routes. We consider one-directional flow going from origins along the feeders' routes towards destinations at the main routes, as might be expected in emergency evacuations or recoveries from major disruptions.

In Section 1 we describe the operations within the observed intermodal system and explain the tradeoff between different types of costs. The anticipated types of costs, which are included in the objective function, are formulated in Section 2. Section 3 explains the constraints, while Section 4 provides the model formulation that is further tested on numerical examples designed in Section 5. Finally we draw conclusions and suggest possible extensions of this work.

\section{PROBLEM}

We consider an intermodal system with relatively short truck routes that feed cargo to major airplane routes (Fig. 1), which has suffered a major disruption. In order to reduce the backlogs accumulated along the feeder routes while the system is inoperative, each truck operates nonstop and fully loaded between an origin and the hub, without pausing between such round trips while backlogs persist. The trucks collect freight from multiple origins along their feeder routes and deliver it at the airport hub. When the takeoff on route is scheduled at time, the airplane is filled to capacity with freight, as long as freight backlogs persist. If the airplane cannot carry all the freight waiting at the airport, the remaining freight has to wait for the next flight with available capacity. On the other hand, if prior to the takeoff, there is little freight in the terminal's storage connecting to route $l$, the airplane's capacity is underused and an additional flight may be needed later. For simplicity, we assume that all trucks are similar and all operate at equal maximum capacity. Moreover, we assume that airplanes have similar capacities. Finally, we assume that the expected amount of cargo waiting for connections can never exceed a preset multiple (e.g. 0.8) of the terminal's storage capacity.

Our objective is to find the optimal (i) number of takeoffs on each air route and (ii) corresponding schedule, for the given probabilistic durations of roundtrips on truck routes. In computing total cost we consider the storage cost, in-terminal operation cost, penalty for late delivery, and airline service cost. A tradeoff exists between the aforementioned types of costs. The earlier one schedules the takeoff, the lower are the storage and penalty costs associated with the freight that successfully connects. However, the earlier the takeoff is scheduled, the greater are the chances that an airplane's capacity will be underused due to insufficient level of stock. Operating less than full airplanes may require running additional flights, thereby increasing the airline service cost.

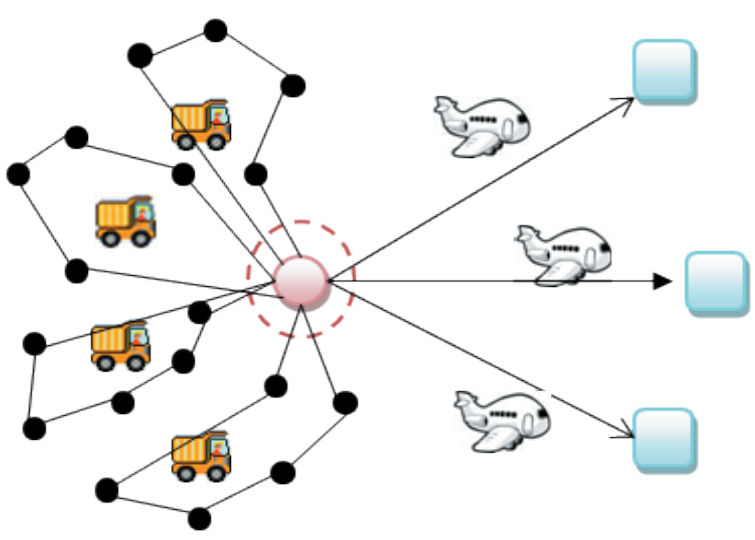

Fig. 1. Intermodal freight system

\section{COSTS}

In this section we introduce the notation used and explain how various types of costs are computed. We begin with the assumptions that allow us to model the arrivals on feeder routes as a Poisson process. We then compute the arrival intensities, which are further used in the development of storage, in-terminal operation, penalty, and airline cost.

Suppose that a single truck operates on a relatively short feeder route $i$ whose starting and end point is the terminal where the truckload connects to the airplane route $l$. Let's assume that the duration of the truck's roundtrip is exponentially distributed with a mean denoted as $1 / \lambda_{i}^{l}$. Moreover, it is reasonable to assume that the observed transportation process has the following three properties:

1. The probability that a truck will accomplish more than one roundtrip within an infinitesimal time interval is negligible.

2. The duration of a roundtrip does not depend on the duration of the previously completed roundtrip.

3. The probability that a roundtrip will end within the time interval $t$ depends on the interval's length, rather than on the time period in which $t$ was observed.

Having adopted the above assumptions, we can model the truck arrivals as a Poisson process with the mean arrival rate $\lambda_{i}^{l}$ according to [9] and [10]. If we assign to feeder route $i$ more than one truck, the 
arrival rate on route $i$ is given in Eq. (1), in which $n_{i}$ represents the number of trucks assigned to feeder route $i$.

$$
r_{i}=n_{i} \lambda_{i}^{l}
$$

Furthermore, if we define with $I l$ the set of feeder routes connecting to main route $l$, the arrival rate of truckloads connecting to route $l$ is:

$$
\lambda^{l}=\sum_{i \in I^{l}} n_{i} \lambda_{i}^{l} .
$$

If we denote the $j^{\text {th }}$ takeoff time on route $l$ as $t_{j}^{l}$, the expected number of truckloads connecting to route $l$ and arriving to terminal between two consecutive flights is:

$$
E\left[R^{l}\left(t_{j}^{l}-t_{j-1}^{l}\right)\right]=\lambda^{l}\left(t_{j}^{l}-t_{j-1}^{l}\right)
$$

\subsection{Storage Cost}

To compute the storage cost, we need to keep track of the inventory level. Moreover, since multiple feeder routes connect to multiple main routes, we need to know the stock for each main route. Therefore we define the variable $S_{j}^{l}$, which defines the inventory level of freight connecting to main route $l$, after the $j^{\text {th }}$ takeoff. We also define $A_{j}^{l}$ representing the amount of freight transported in the $j^{\text {th }}$ flight on route $l$. Considering the inflow and outflow of freight into the terminal storage, Eq. (4) has to hold for all flights on all routes. Please note that $S_{0}^{l}, t_{0}^{l}$ and $A_{0}^{l}$ all equal 0 . Moreover, we assume that all the freight arriving at the terminal before the last scheduled takeoff has to be flown. Thus we also set $S_{n^{l}}^{l}$ to equal 0 .

$$
S_{j-1}^{l}+\lambda^{l}\left(t_{j}^{l}-t_{j-1}^{l}\right)=A_{j}^{l}+S_{j}^{l} \forall j \in J^{l} \quad \forall l \in L .
$$

Moreover, since we do not know in advance if there will be enough freight in the terminal's storage to fill the airplane, we specify in Eq. (5) that the airplane will be loaded with all the connecting freight found in terminal that can fit within the airplane's capacity, denoted $A_{c}$.

$$
A_{j}^{l}=\max \left\{A_{c}, S_{j-1}^{l}+\lambda^{l}\left(t_{j}^{l}-t_{j-1}^{l}\right)\right\} .
$$

Based on the previous derivations, in Eq. (6) we can compute the storage cost between two consecutive flights for freight connecting to route $l$. Please note that $C_{D T}$ denotes the storage cost per truckload-hour.

$$
\frac{1}{2}\left(S_{j-1}^{l}+S_{j}^{l}\right)\left(t_{j}^{l}-t_{j-1}^{l}\right) C_{D T} .
$$

We can further compute the total storage cost for freight connecting to route $l$ by summing Eq. (6) over all the flights in $J$.

$$
S C^{l}=\frac{1}{2} \sum_{j \in J^{l}}\left(S_{j}^{l}+S_{j-1}^{l}\right)\left(t_{j}^{l}-t_{j-1}^{l}\right) C_{D T} .
$$

Finally, we can compute the total storage cost by summing Eq. (7) over the set $L$, which denotes main routes.

$$
S C=\frac{1}{2} \sum_{l \in L} \sum_{j \in J^{l}}\left(S_{j}^{l}+S_{j-1}^{l}\right)\left(t_{j}^{l}-t_{j-1}^{l}\right) C_{D T} .
$$

\subsection{In-terminal Operation Cost}

Here we analyse the loading and unloading cost due to the cargo transfer from trucks to airplanes. We assume that the in-terminal operation cost is lower when a truck arrives slightly before the takeoff and takes its truckload directly to the airplane, instead of unloading it in the terminal storage. Therefore, let's define parameter $d$ so that a truck arriving within the $\left(t_{j}^{l}-d, t_{j}^{l}\right)$ interval takes its truckload directly to the airplane. Now we can compute the expected number of truckloads that will be loaded directly on the airplane:

$$
b_{d}=\sum_{l \in L} \sum_{j \in J^{l}} \lambda^{l} d .
$$

Here we assume that $d$ is smaller than the interval between two consecutive flights on the same route. Thus, the expected number of truckloads being loaded directly onto the airplane depends on the number of takeoffs rather than on their schedule.

If we denote $C_{t d}$ to be the unit in-terminal operation cost for the case of direct transfer to the airplane, $C_{t i}$ to be the unit cost for the case of indirect transfer to the airplane, and $G$ to be the total number of truckloads, then the total in-terminal operation cost is:

$$
I C=b_{d} C_{t d}+\left(G-b_{d}\right) C_{t i} .
$$

\subsection{Penalty Cost}

A penalty is imposed for late delivery, reflecting the lower value of freight that is delivered later. Here we assume that the time of the takeoff is relevant 
for computing the penalty cost. We define a penalty function $f_{p}$ as the piecewise linear function of takeoff time starting from the beginning of the observed time period (the moment the system starts recovering from a disruption), as shown in Fig. 2.

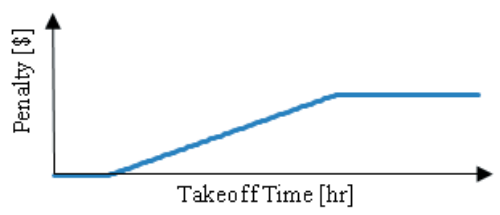

Fig. 2. Penalty function $f_{p}$

Now we can compute the penalty cost by summing the penalty for all the flights on all the air routes, as shown in Eq. (11). Please note that we again use $A_{j}^{l}$ as defined in Eq. (5), which denotes the number of truckloads carried on the $j^{\text {th }}$ takeoff on route $l$.

$$
P C=\sum_{l \in L} \sum_{j \in J^{l}} A_{j}^{l} f_{p}\left(t_{j}^{l}\right) .
$$

\subsection{Airline Cost}

The last type of cost considered is the airline service cost, which covers the use of both airplanes and airport facilities. It is proportional to the number of the airplane roundtrips (takeoffs). We denote the number of takeoffs on route $l$ as $n^{l}$. Moreover, we denote as $C_{A}^{l}$ the cost of an airplane roundtrip on route $l$. Finally, the total airline service cost is:

$$
A C=\sum_{l \in L} n^{l} C_{A}^{l}
$$

\section{CONSTRAINTS}

In this section we analyse several constraints needed in order for the mathematical model to fairly represent the real world. The first constraint that we consider is the time window constraint for takeoffs. Utilisation of airport facilities is often restricted to certain time slots. Thus each takeoff must be scheduled within a prespecified time interval. Therefore, the time window constraint is:

$$
a_{j}^{l}<t_{j}^{l}<b_{j}^{l} \forall j \in J^{l} \quad \forall l \in L .
$$

Since limited airport capacity might require a minimum time interval between any two flights, we introduce the following constraint.

$$
t_{j}^{l}-t_{j-1}^{l} \geq t_{\min } \quad \forall j \in J^{l} \quad \forall l \in L .
$$

The last constraint we consider is the terminal's storage capacity. We assume that the expected amount of freight at the terminal should never exceed the multiple $m_{s}$ of the storage capacity $S_{c}$. Since we previously explained how the expected inventory level for freight connecting to route $l$ at takeoff time $t_{j}^{l}$ is computed, we must now ensure that the total expected inventory never exceeds the storage capacity $m_{s} S_{c}$. Thus we define parameter $p_{t}$ and control the total inventory level at time $p_{t}$. In order to do so we first need to find the inventory level of freight connecting to route $l$ at time $p_{t}$. We introduce $s^{l}$ which denotes the takeoff time on route $l$ prior to $p_{t}$ and define $k^{l}$ which equals the takeoff index $j$.

$$
\begin{gathered}
s^{l}=\max \left\{t_{j}^{l}: t_{j}^{l} \leq p_{t}\right\}, \\
k^{l}=\text { index } j:\left(t_{j}^{l} \leq p_{t} \wedge t_{j+1}^{l} \geq p_{t}\right) .
\end{gathered}
$$

Now we can compute the expected inventory of freight connecting to route $l$ as:

$$
S_{k^{l}}^{l}+\lambda^{l}\left(p_{t}-s^{l}\right)
$$

Finally we can define the storage capacity constraint by summing Eq. (17) over the set of main routes and setting the sum below the storage capacity $S_{c}$ multiplied by $m_{s}$ (a safety factor). Please note that the constraint in Eq. (18) should hold for any real value of time parameter $p_{t}$.

$$
\sum_{l \in L} S_{k^{l}}^{l}+\lambda^{l}\left(p_{t}-s^{l}\right) \leq S_{c} m_{s} \quad \forall p_{t} \in R .
$$

\section{MODEL}

In the previous section, the types of costs and constraints considered were explained. Now we can present the mathematical formulation of the model in Eqs. (19) to (30), which represents a nonlinear program. Here we provide a compact formulation of the objective function using Eqs. (8), and (10) to (12). In Eqs. (20) to (30), we provide the constraints and other previously derived relationships.

$$
\operatorname{Min} T C=S C+I C+P C+A C,
$$

subject to:

$$
S_{j-1}^{l}+\lambda^{l}\left(t_{j}^{l}-t_{j-1}^{l}\right)=A_{j}^{l}+S_{j}^{l} \forall j \in J^{l} \quad \forall l \in L,
$$




$$
\begin{gathered}
S_{j-1}^{l}+\lambda^{l}\left(t_{j}^{l}-t_{j-1}^{l}\right)=A_{j}^{l}+S_{j}^{l} \forall j \in J^{l} \forall l \in L, \\
a_{j}^{l}<t_{j}^{l}<b_{j}^{l} \forall j \in J^{l} \forall l \in L, \\
t_{j}^{l}-t_{j-1}^{l} \geq t_{\min } \forall j \in J^{l} \forall l \in L, \\
\sum_{l \in L} S_{k^{l}}^{l}+\lambda^{l}\left(p_{t}-s^{l}\right) \leq S_{c} m_{s} \forall p_{t} \in R, \\
A_{j}^{l}=\max \left\{A_{c}, S_{j-1}^{l}+\lambda^{l}\left(t_{j}^{l}-t_{j-1}^{l}\right)\right\}, \\
s^{l}=\max _{j}\left\{t_{j}^{l}: t_{j}^{l} \leq p_{t}\right\} \\
k^{l}=i n d e x j:\left(t_{j}^{l} \leq p_{t}^{\wedge} t_{j+1}^{l} \geq p_{t}\right), \\
b_{d}=\sum_{l \in L} \sum_{j \in J^{l}} \lambda^{l} d, \\
S_{0}^{l}=t_{0}^{l}=A_{0}^{l}=S_{n^{l}}^{l}=0, \\
t_{j}^{l} \in R_{+} \forall j \in J^{l} \forall l \in L, \\
n^{l} \in Z_{+} \forall l \in L .
\end{gathered}
$$

The total cost function is a function of the number of takeoffs and takeoff times, as explained in the problem statement. The nonlinear model shown in Eqs. (19) to (30) optimises the schedule while taking into consideration the capacity of airplanes, airport and terminal storage, and time windows for takeoffs. In the following section we apply a genetic algorithm (GA) to optimise the schedule in two case studies. Interested readers may refer to [11] and [12] for more information about GA's.

\section{APPLICATION}

In order to test our model, we designed two case studies. In the first case, the schedule in an intermodal system with a single main route is optimised. In this simplified optimisation problem we examine the anticipated tradeoff in types of costs through sensitivity analysis. In the second case we analyse a complex system with multiple main routes and time windows.

\subsection{Case Study with a Single Air Route}

We analyse a system with ten feeder truck routes connecting to a single airplane main route. In Table 1 we provide the average roundtrip time on each feeder route, as well as the number of vehicles operating on each truck route. We seek to optimise the number of takeoffs and corresponding schedule assuming that all the freight arriving at the terminal before the last takeoff has to be transported. For this case, we assume the input data from Table 2. The optimisation results for 4 to 9 takeoffs are presented in Table 3. We present an optimised schedule for six different numbers of takeoffs and corresponding costs in dollars. Please note that within "other costs" we consider storage, penalty, loading and unloading costs. Moreover, by marginal savings in other costs we consider savings in storage, penalty, loading and unloading costs due to introducing an additional roundtrip flight.

Table 1. Vehicle size and roundtrip duration

\begin{tabular}{ccc}
\hline $\begin{array}{c}\text { Feeder } \\
\text { route }\end{array}$ & $\begin{array}{c}\text { Average roundtrip } \\
\text { duration } 1 / \lambda_{1}[\mathrm{hr}]\end{array}$ & $\begin{array}{c}\text { Number of trucks } \\
\text { in feeder route }\end{array}$ \\
\hline 1 & 1.23 & 3 \\
\hline 2 & 1.97 & 4 \\
\hline 3 & 1.73 & 1 \\
\hline 4 & 2.10 & 2 \\
\hline 5 & 2.16 & 1 \\
\hline 6 & 1.94 & 2 \\
\hline 7 & 2.18 & 5 \\
\hline 8 & 1.86 & 2 \\
\hline 9 & 1.68 & 1 \\
\hline 10 & 2.00 & 3 \\
\hline
\end{tabular}

The results presented in Table 3 show that the minimum total cost occurs in the case with five takeoffs. Therefore we can conclude that at the cost of $7000 \$$ /flight, one more flight than necessary to satisfy the demand should be introduced. Moreover, we can observe that storage, penalty and loading/ unloading cost decrease with the increase in the number of takeoffs. This outcome was expected and it confirmed the tradeoff between types of cost that was explained in the problem statement. We also note that the marginal savings in storage, penalty and loading/ unloading cost decreases with the number of aircraft roundtrips, which is another anticipated outcome.

Based on the values for storage, penalty and loading/unloading cost we can explore how different flight costs affect the optimised number of takeoffs and thereby the schedule. In Fig. 3, we plot total cost for the case of 4, 5, 6, 7, 8 and 9 roundtrips vs. aircraft roundtrip cost. Fig. 3 also shows five threshold values for airplane roundtrip cost which determine the optimal number of takeoffs. Those values are 1357, 1871, 2771, 4277 and 7509 dollars, respectively. Clearly, for a relatively low cost per plane roundtrip, the total system cost is optimised by scheduling more takeoffs than necessary to satisfy the demand. As the 
Table 2. Input data

\begin{tabular}{|c|c|c|c|c|c|}
\hline Airplane capacity & $A_{c}$ & 50 truckloads & In-terminal cost & $C_{t d}$ & 25 \$/truckload \\
\hline Flight cost & $C_{A}^{1}$ & 7,000 \$/roundtrip & In-terminal cost & $c_{t i}$ & 45 \$/truckload \\
\hline Terminal storage capacity & $S_{c}$ & 87.5 truckloads & Time of the last takeoff & $t_{n}^{1}$ & $15 \mathrm{hrs}$ \\
\hline Storage multiple (safety factor) & $m_{s}$ & 0.8 & Minimum headway & $t_{\min }$ & $0.8 \mathrm{hrs}$ \\
\hline Storage cost & $C_{D T}$ & 4 \$/truckload-hr & Arrival rate based on the data from Table 1 & $\lambda^{1}$ & $12.95 \mathrm{veh} / \mathrm{hr}$ \\
\hline Amount of time & $d$ & $15 \min$ & Penalty function & $f_{p}(t)$ & $\begin{array}{l}0 \text { if } t \leq 2 \\
125 t-250 \text { if } 2<t \leq 10 \\
1000 \text { if } t>10\end{array}$ \\
\hline
\end{tabular}

Table 3. Optimised Schedules and Costs

\begin{tabular}{|c|c|c|c|c|c|c|c|c|c|c|c|c|c|}
\hline \multirow{2}{*}{$\begin{array}{l}\text { Number } \\
\text { of } \\
\text { flights }\end{array}$} & \multirow{2}{*}{$\begin{array}{l}\text { Airline } \\
\text { cost } \\
{\left[10^{3} \$\right]}\end{array}$} & \multirow{2}{*}{$\begin{array}{c}\text { Other } \\
\text { cost } \\
{\left[10^{3} \$\right]}\end{array}$} & \multirow{2}{*}{$\begin{array}{c}\text { Marginal } \\
\text { savings } \\
\text { in other } \\
\text { cost }\end{array}$} & \multirow{2}{*}{$\begin{array}{c}\text { Total } \\
\text { cost } \\
{\left[10^{3} \$\right]}\end{array}$} & \multicolumn{9}{|c|}{$\begin{array}{c}\text { Optimised takeoff times } \\
\text { for the given number of flights [hr] }\end{array}$} \\
\hline & & & & & Fl.1 & Fl.2 & Fl.3 & Fl.4 & Fl.5 & Fl.6 & Fl. 7 & Fl.8 & Fl.9 \\
\hline 4 & 28 & 151 & NA & 179 & 3.6 & 7.3 & 11.1 & 15.0 & NA & NA & NA & NA & NA \\
\hline 5 & 35 & 143 & 8 & 178 & 2.5 & 5.0 & 7.5 & 11.3 & 15.0 & NA & NA & NA & NA \\
\hline 6 & 42 & 139 & 4 & 181 & 2.0 & 4.0 & 6.0 & 8.0 & 11.5 & 15.0 & $\mathrm{NA}$ & NA & NA \\
\hline 7 & 49 & 136 & 3 & 185 & 2.0 & 3.6 & 5.2 & 6.8 & 8.4 & 11.7 & 15.0 & NA & NA \\
\hline 8 & 56 & 134 & 2 & 190 & 2.0 & 3.3 & 4.7 & 6.0 & 7.4 & 8.7 & 11.9 & 15.0 & NA \\
\hline 9 & 63 & 133 & 1 & 196 & 2.0 & 3.2 & 4.3 & 5.5 & 6.6 & 7.8 & 8.9 & 12.0 & 15.0 \\
\hline
\end{tabular}

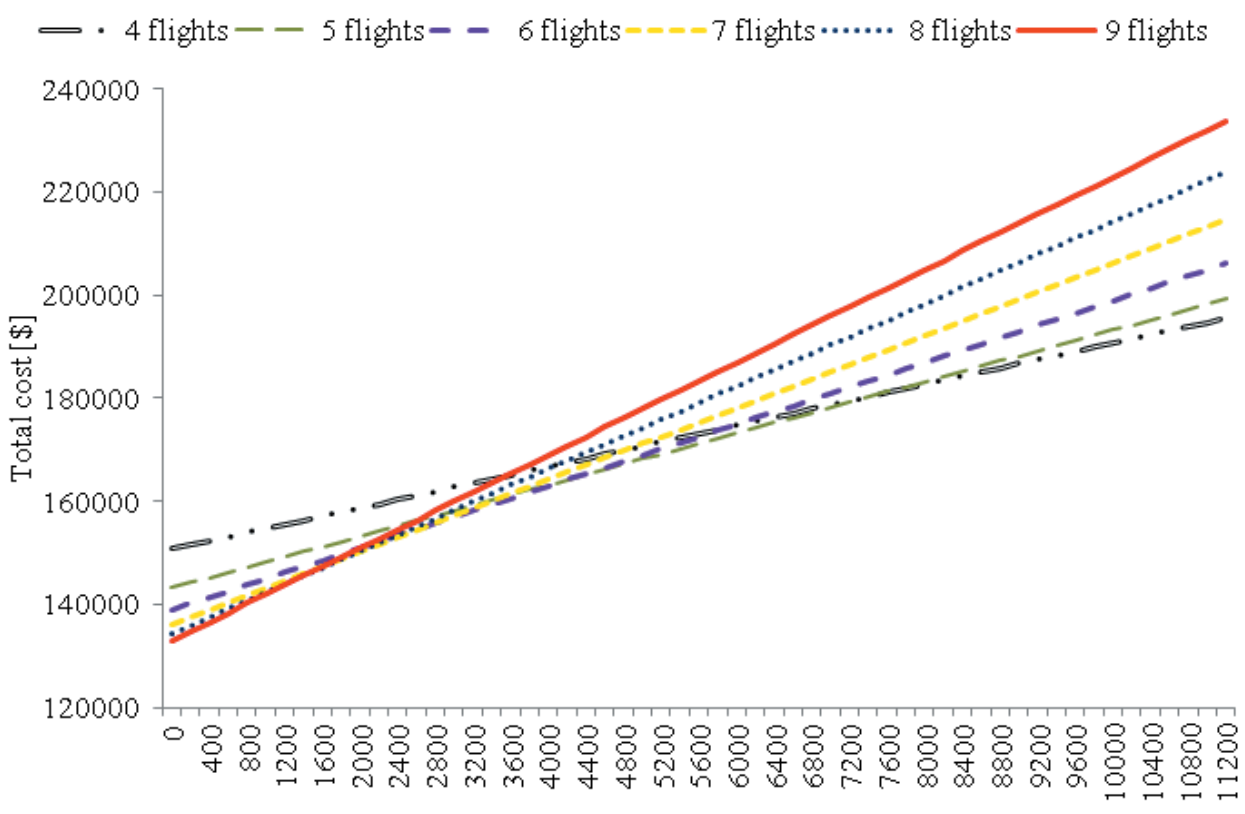

Flight cost [\$]

Fig. 3. Sensitivity Analysis

airline cost increases, the optimal number of takeoffs decreases until it eventually drops to the minimum number needed to satisfy the demand.

\subsection{Case Study with Multiple Airline Routes}

Here we consider the case of multiple feeder routes connecting to three air routes of similar lengths. Since 
we consider the case with three dozen feeder routes, we do not present the expected roundtrip duration and the number of vehicles on each route, as we did in the previous example. Instead, we provide the computed arrival rates of vehicles connecting to three air routes. Moreover, we assume the same values from the previous numerical example, but this time we include time windows into our analysis. The aforementioned data are provided in Table 4. Please note again that, for each route, all the freight arriving at the terminal by the time of the last flight has to be loaded into airplanes and transported to its destination.

Table 4. Input data

\begin{tabular}{lll}
\hline Flight cost on all routes & $C_{A}^{1}$ & $9,000 \$$ \\
\hline Arrival rate on route 1 & $\lambda^{1}$ & $8.0 \mathrm{veh} / \mathrm{hr}$ \\
\hline Arrival rate on route 2 & $\lambda^{2}$ & $8.5 \mathrm{veh} / \mathrm{hr}$ \\
\hline Arrival rate on route 3 & $\lambda^{3}$ & $7.5 \mathrm{veh} / \mathrm{hr}$ \\
\hline Time windows for Route 1 & $\left(a_{1}^{1}, b_{1}^{1}\right)$ & $(1.5,2.5)$ \\
\cline { 2 - 3 } & $\left(a_{2}^{1}, b_{2}^{1}\right)$ & $(3.1,4.5)$ \\
\hline Time windows for Route 2 & $\left(a_{1}^{2}, b_{1}^{2}\right)$ & $(1.2,3.6)$ \\
\cline { 2 - 3 } & $\left(a_{2}^{2}, b_{2}^{2}\right)$ & $(4.5,6.4)$ \\
\cline { 2 - 3 }$\left(a_{3}^{2}, b_{3}^{2}\right)$ & $(7.0,10.0)$ \\
\hline Time windows for Route 3 & $\left(a_{1}^{3}, b_{1}^{3}\right)$ & $(2.3,4.2)$ \\
\cline { 2 - 3 } & $\left(a_{2}^{3}, b_{2}^{3}\right)$ & $(5.2,6.5)$ \\
\hline Minimum time interval between & $t_{\min }$ & 0.5 hours \\
\hline any two flights & $t_{n}^{1}$ & 15 hours \\
\hline Last takeoff on Route 1 & $t_{n}^{2}$ & 14 hours \\
\hline Last takeoff on Route 2 & $t_{n}^{3}$ & 16 hours \\
\hline Last takeoff on Route 3 & &
\end{tabular}

Table 5. Optimised cost

\begin{tabular}{ccccc}
\hline \multirow{2}{*}{ Case } & \multicolumn{3}{c}{ Number of Flights on Route I } & Total Cost \\
\cline { 2 - 4 } & $\mathrm{I}=1$ & $\mathrm{I}=2$ & $\mathrm{I}=3$ & {$[103 \$$} \\
\hline 1 & 3 & 3 & 3 & $\mathrm{NA}$ \\
\hline 2 & 4 & 3 & 3 & $\mathrm{NA}$ \\
\hline 3 & 3 & 4 & 3 & $\mathrm{NA}$ \\
\hline 4 & 3 & 3 & 4 & $\mathrm{NA}$ \\
\hline 5 & 4 & 4 & 4 & 378 \\
\hline 6 & 4 & 4 & 5 & 387 \\
\hline 7 & 4 & 5 & 4 & 386 \\
\hline 8 & 5 & 4 & 4 & 384 \\
\hline 9 & 5 & 5 & 4 & 390 \\
\hline 10 & 5 & 4 & 5 & 389 \\
\hline 11 & 4 & 5 & 5 & 394 \\
\hline 12 & 5 & 5 & 5 & 396 \\
\hline
\end{tabular}

The optimization results for the case study with three main routes are given in Table 5. NA stands for the cases when no feasible solution is found either due to overloading of the terminal storage or due to not delivering all the freight that has arrived by the time of the last takeoff on each air route.

From Table 5 we conclude that at 9000 \$/flight, the total cost is optimised by scheduling 4 flights on each main route (case 5), which also equals the minimum number of flights needed to provide a feasible solution. Finally, in Table 6 we provide the optimised schedule for all 8 feasible combinations of flights from Table 5 .

Table 6. Optimised schedule

\begin{tabular}{|c|c|c|}
\hline Case & Route & Optimised Schedule on Route \\
\hline \multirow{3}{*}{5} & 1 & $2.19|4.50| 8.75 \mid 15.00$ \\
\hline & 2 & $2.69|5.51| 8.12 \mid 14.00$ \\
\hline & 3 & 3.19 | 6.26 | $9.33 \mid 16.00$ \\
\hline \multirow{3}{*}{6} & 1 & $2.19|4.50| 8.75 \mid 15.00$ \\
\hline & 2 & $2.69|5.40| 8.12 \mid 14.00$ \\
\hline & 3 & $3.19|6.22| 9.25|12.63| 16.00$ \\
\hline \multirow{3}{*}{7} & 1 & $2.17|4.50| 8.75 \mid 15.00$ \\
\hline & 2 & $2.69|5.12| 7.57|10.79| 14.00$ \\
\hline & 3 & 3.17 | $6.25|9.33| 16.00$ \\
\hline \multirow{3}{*}{8} & 1 & $2.19|4.50| 7.27|11.13| 15.00$ \\
\hline & 2 & $2.69|5.40| 8.12 \mid 14.00$ \\
\hline & 3 & 3.19 | 6.26 | $9.33 \mid 16.00$ \\
\hline \multirow{3}{*}{9} & 1 & $2.15|4.43| 6.71|8.73| 15.00$ \\
\hline & 2 & $2.65|5.04| 7.44 \mid 9.83 \quad 14.00$ \\
\hline & 3 & 3.15 | $6.21|9.33| 16.00$ \\
\hline \multirow{3}{*}{10} & 1 & 2.19 | 4.37 | $6.56|8.75| 15.00$ \\
\hline & 2 & $2.69|5.34| 8.12 \mid 14.00$ \\
\hline & 3 & $3.19|5.84| 7.62|11.81| 16.00$ \\
\hline \multirow{3}{*}{11} & 1 & $2.18|4.50| 8.75 \mid 15.00$ \\
\hline & 2 & $2.68|5.04| 7.40 \mid 9.75 \quad 14.00$ \\
\hline & 3 & $3.18|6.21| 9.25|12.63| 16.00$ \\
\hline \multirow{3}{*}{12} & 1 & $2.01 \mid 4.27$ | $6.51 \mid 8.75 \quad 15.00$ \\
\hline & 2 & $2.51|4.77| 7.01|8.25| 14.00$ \\
\hline & 3 & $3.01 \mid 5.37$ | 7.72 | $11.86 \mid 16.00$ \\
\hline
\end{tabular}

\section{CONCLUSIONS}

This paper studied the recovery of a single-terminal intermodal freight system from a disruption. A model was developed that optimises the schedule of vehicles on main routes assuming Poisson arrivals on feeder routes. A genetic algorithm was used to optimise several case studies and sensitivity analysis confirmed 
the anticipated tradeoff in types of cost. Moreover, the model developed in this paper was applied to case studies including feeder truck routes and main airline routes. However, this model could be applied to other combinations of transportation modes without many modifications, as long as the arrivals do not deviate much from Poisson distributions.

Since the arrivals were modeled as a Poisson process, the computational efficiency of the model is fairly insensitive to the number of feeder routes or operating vehicles. Therefore the proposed scheduling model can be successfully applied to optimise the performance of busy intermodal systems with numerous vehicle arrivals.

Several assumptions built into this paper may be relaxed in the future in order to make the model more general. The current model could be improved to provide good robust solutions even for the case when some of the three properties of the Poisson process listed in Section 1 do not hold. Moreover, the current analysis assumes fixed numbers of vehicles operating on the feeder routes. Future work may consider variable fleet sizes on feeder routes and thereby nonstationary arrival intensities.

\section{NOTATION}

The following symbols are used in this paper:

$\lambda_{i}^{l}$ parameter of the exponentially distributed duration of truck roundtrip on feeder route $i$ connecting to main route $l$

$i \quad$ index of feeder route

$l \quad$ set of feeder routes

$I^{l} \quad$ set of feeder routes connecting to main route $l$; clearly $I^{l} \in I$

$j \quad$ index of takeoffs on route $l$

$J l \quad$ set of takeoffs on route $l$

$l$ index of main route

$k^{l} \quad$ index of the takeoff on route $l$ prior to time $p_{t}$

$L$ set of main routes

$t_{j} \quad$ time of the $j^{\text {th takeoff on route } l}$

$n^{l}$ number of takeoffs on main route $l$

$n_{i} \quad$ number of trucks on feeder route $i$

$r_{i}$ arrival rate on feeder route $i$

$A_{c}$ capacity of an airplane

$S_{c}$ capacity of terminal's storage

$m_{s}$ storage multiple

$C_{D T}$ in-terminal dwell cost

$C_{A}^{l}$ flight cost on route $l$

$\mathrm{SC}^{l}$ storage cost associated with freight connecting to main route $l$

$S C$ storage cost $d$ the amount of time such that a truck arriving within the $\left(t_{j}-d, t_{j}\right)$ interval will take its truckload directly to the airplane

$S_{j}^{l}$ inventory level of freight connecting to main route $l$ after the $j^{\text {th }}$ takeoff

$b_{d}$ the expected number of truckloads that will be transferred directly from trucks to airplanes

$\mathrm{C}_{t i}$ cost of in-terminal operations

$\mathrm{C}_{t d}$ cost of in-terminal operations when the truck takes its truckload directly to the airplane

IC overall cost for in-terminal operations

$f_{p}\left(t_{j}^{l}\right)$ penalty function per truckload loaded into airplane at moment $t_{j}^{l}$

$P C$ overall penalty cost

$A C$ overall airline cost

$T C$ total cost

$t_{\min }$ minimum time interval between any two takeoffs

$a_{j}^{l}$ the lower bound for the $j^{\text {th theoff on route } l}$

$b_{j}^{l}$ the upper bound for the $j^{\text {th }}$ takeoff on route $l$

$A_{j}^{l}$ amount of freight carried in the $j^{\text {th }}$ takeoff on route $l$

$p_{t}$ control parameter used to check the inventory level

$R_{+}$set of nonnegative real numbers

$Z_{+}$set of nonnegative integers

\section{ACKNOWLEDGMENTS}

This work was sponsored by the U. S. Department of Transportation (USDOT) and the Maryland State Highway Administration (MSHA). The authors thank the Center for Integrated Transportation Systems Management (CITSM) of the University of Maryland for its support.

\section{REFERENCES}

[1] Li, W., Glazebrook, D.K. (1998). On stochastic machine scheduling with general distribution assumptions. European Journal of Operations Research, vol. 105, no. 3, p. 525-536, DOI:10.1016/S0377-2217(97)00088-X.

[2] Petkov, S., Maranas, C. (1997). multiperiod planning and scheduling of multiproduct batch plants under demand uncertainty. Industrial \& Engineering Chemistry Research, vol. 36, p. 4864-4881, DOI:10.1021/ie970259z.

[3] Pundoor, G., Chen, Z.-L. (2009). Joint cyclic production and delivery scheduling in a two-stage supply chain. International Journal of Production Economics, vol. 119, no. 1, p. 55-74, DOI:10.1016/j.ijpe.2009.01.007.

[4] Ting, C.J., Schonfeld, P. (2005). Schedule coordination in a multiple terminal transit network. Journal of Urban Planning and Development, vol. 131, no. 2, p. 112-124, DOI:10.1061/(ASCE)0733-9488(2005)131:2(112). 
[5] Chen, C.C., Schonfeld, P. (2010). Modeling and performance assessment of intermodal transfers at cargo terminals. Transportation Research Record: Journal of the Transportation Research Board, vol. 2162, no. 1, p. 53-62, DOI:10.3141/2162-07.

[6] Teodorović, D. (1988). Simultaneous determining departure time and flight frequency on a route. Airline Operations Research, Gordon and Breach Science, New York, p. 131-145.

[7] Chang, S.W., Schonfeld, P. (2004). Optimized schedules for airline routes. Journal of Transportation Engineering, ASCE, vol. 130, no. 4, p. 412-418, DOI:10.1061/(ASCE)0733-947X(2004)130:4(412).

[8] Marković, N., Schonfeld, P. (2011). Scheduling under uncertainty in a single-hub intermodal freight system.
Transportation Research Record: Journal of the Transportation Research Board, vol. 2238, no.1, p. 2431, DOI:10.3141/2238-04.

[9] Vukadinović, S. (1988). Queuing, Naučna Knjiga, Beograd. In Serbian

[10] Wolff, R. (1989). Stochastic modeling and the theory of queues. Prentice-Hall, Englewood Cliffs.

[11] Goldberg, D.E. (1995). Genetic algorithms in search, optimization and machine learning. Addison Wesley, Boston,.

[12] Michalewicz, Z. (1996). Genetic algorithms + data structure $=$ evolution programs, 3rd ed., SpringerVerlag, London, DOI:10.1007/978-3-662-03315-9. 